David S. Warner MD

\title{
Anesthesia for craniotomy
}

I T is not surprising that anesthesia for craniotomy presents special considerations. The brain is enclosed in a rigid skull and the majority of craniotomies are performed for the treatment of space occupying lesions. At the same time, the brain is a highly vascular organ presenting potential for massive perioperative hemorrhage. Tolerance of the brain to interruption of substrate delivery is minimal.

Anesthetics and physiologic factors controlled by the anesthesiologist have profound effects on the brain. Interactions between anesthesia and surgical outcome can be expected. This article is intended as a practical review of the anesthetic management of patients with intracranial pathology requiring surgery.

\section{Preoperative evaluation}

The initial approach to the patient requiring craniotomy is similar to that of all other patients. There are several additional considerations. It is important to obtain an appropriate baseline neurologic evaluation. At emergence from anesthesia, failure to recover baseline neurologic function can be attributed to surgery, anesthesia, or an interaction between the two. It is incumbent on the anesthesiologist to recognize changes from baseline so as to participate in making the diagnosis. It is also important to gain insight into the magnitude of intracranial hypertension and possible interactions with anesthetic agents. Acute changes in intracranial pressure (ICP; e.g., epidural hematoma) are potentially more devastating and are likely to be more sensitive to anesthetic effects. The anesthesiologist also can benefit from appreciating the characteristics of the lesion with respect to potential for major hemorrhage.

With respect to vascular lesions, it is valuable to know the interval since aneurysmal hemorrhage with respect to urgency for surgery relative of risk of vasospasm. Patients receiving nimodipine/nicardipine may exhibit exaggerated hemodynamic responses to volatile anesthetics. For arteriovenous malformations (AVM), history of preoperative neuroradiologic intervention can assist the anesthesiologist in anticipating magnitude of blood loss and potential for periopera- tive malignant brain swelling. Resection of a radiologically obliterated AVM typically poses little risk for these complications, while previously untreated lesions raise concern. The size of the AVM is likely to be relevant as well.

\section{Monitoring}

For most craniotomies, monitoring consists of standard monitors in addition to an intra-arterial catheter. The arterial catheter is valuable in providing strict control of blood pressure (particularly during emergence). Central venous pressure (CVP) monitoring is usually not required for management of tumours unless the case is expected to be exceedingly long or if major hemorrhage is expected (e.g., vascular meningioma, tumour encasement of major vessels). Otherwise, indications for CVP and pulmonary artery pressure monitoring remain the same as for other patient populations dictated principally by cardiac, renal, and pulmonary status.

One exception to this rule is intracranial aneurysm surgery. This author places CVP monitors in all such patients. Use of mannitol essentially voids urine output as a monitor of intravascular volume status. The brain receives approximately $20 \%$ of cardiac output when the body is at rest. If cardiac output is approximately 5 $\mathrm{L} \cdot \mathrm{min}^{-1}$, it is easy to appreciate that uncontrolled hemorrhage can result in rapid exsanguination. Resuscitation will be aided by the CVP monitor. An important reason for placement of a central venous catheter is delivery of vasoactive medications. It is sometimes difficult to predict whether the surgeon will request blood pressure to be increased (e.g., during temporary vascular occlusion of the parent vessel) or decreased (e.g., to facilitate clipping or reduce rate of hemorrhage). Delivery of drugs into the central circulation provides the fastest possible onset of action and shortens the feedback loop for dose titration facilitating exquisite control of blood pressure within the desired range.

Routine use of intraoperative electrophysiologic monitoring to detect ischemia remains controversial. Although monitoring of evoked potentials make sense,

\footnotetext{
From the Department of Anesthesiology, Duke University Medical Center, Durham, North Carolina, USA.

Address correspondence to: Dr. David S. Warner, Box 3094, Department of Anesthesiology, Duke University Medical Center, Durham, North Carolina, USA 27710. Phone: 919-684-6633; Fax: 919-684- 6692; E-mail: warne002@mc.duke.edu
} 
there are numerous reports of false positive and false negative readings. Some advocate use of electroencephalographic (EEG) monitoring for the purposes of pharmacologically inducing burst suppression for neuroprotection. Efficacy is unsupported by experimental literature although the risk seems small. Monitoring of cranial nerve function is often employed during posterior fossa procedures. Implications for anesthesia largely pertain to limitation in use of muscle relaxants. Although there is no contraindication to use of relaxants during induction and positioning, it is important to assure recovery of neuromuscular function prior to surgical stimulation of the cranial nerves located in the vicinity of the lesion. Surgery for excision of epileptic foci often requires intraoperative EEG mapping. It is important to appreciate the anticonvulsant effects of the different anesthetics. Most $i v$ and volatile anesthetics suppress EEG activity and may degrade mapping. Conversion to a nitrous/narcotic technique prior to mapping with sufficient time to eliminate agents that suppress EEG activity is commonly practiced. Conversely, small doses of pro-epileptic agents (e.g., methohexital) are occasionally requested to aid in identification of the focus.

Transcranial Doppler (TCD) monitoring may be of value preoperatively in screening for vasospasm. Intraoperative use during craniotomy is cumbersome and unsubstantiated as a modifier of outcome. There is increasing hope that computerized analysis of TCD waveforms may provide useful data on intracranial pressure (ICP) and this could be useful during induction. Clearly TCD can identify complete obstruction to blood flow. More useful information regarding magnitude of ICP allowing calculation of cerebral perfusion pressure is not currently available.

\section{Anesthesia induction}

Concerns unique to induction of anesthesia for craniotomy are principally related to ICP in the case of mass lesions or prevention of hemorrhage in the case of vascular lesions. The history of effects of anesthetics on ICP during induction began in the 1960s when the earliest measurements were made on patients anesthetized for tumour surgery. ${ }^{1}$ Major increases in ICP were observed with anesthetic induction. In the subsequent zeal to provide optimal care, it was felt that any increase in ICP could only be adverse to the patient and thus use of anesthetics known to increase ICP was discouraged. Although logical, this came at some cost. Something must be used to provide anesthesia and those drugs known to reduce ICP (e.g., thiopental) typically have prolonged durations of action or produce hemodynamic instability. In fact, data relevant to effects of various anesthetics on ICP in humans is limited. Most information has been derived from animal studies. More important, there is little data regarding any relationship between anesthetic effects on ICP and outcome from craniotomy. The few human studies that have been performed have used crude outcome assays preventing a definitive definition of the relevance of this problem. As a result, the use of various anesthetics during craniotomy has been broadened to allow all facets of a successful anesthetic to be considered. ${ }^{2}$

We cannot measure ICP in each patient. As a result, we rely on information derived from limited human data and extrapolate information from animal studies. This seems to work. Case reports in the literature showing a causal relationship between anesthetics and brain herniation on induction are almost non-existent. The one exception to this is patients with an occult lesion undergoing surgery for non-neurosurgical procedures. The vast majority of patients anesthetized for craniotomy emerge from anesthesia either with neurologic status unchanged or with changes directly attributable to the site of surgery. As a result, it is difficult to advocate any specific anesthetic or technique for the purpose of induction. We do know that ICP effects of volatile anesthetics can be blunted by simultaneous moderate hyperventilation. ${ }^{3}$ We also know that high concentrations of volatile anesthetics perturb cerebral autoregulation. ${ }^{4}$ We also know that there are numerous methods to blunt hemodynamic responses to endotracheal intubation and application of the pin head-holder. Cumulatively these concerns must be weighed when inducing anesthesia for craniotomy.

With respect to cerebral aneurysms, ICP is of less concern than is prevention of abrupt and major increases in mean arterial pressure (MAP) that may contribute to rupture of the lesion. In this case, there is abundant evidence that a poorly controlled hemodynamic state during induction is contributory. The mortality rate associated with aneurysmal rupture during induction is substantial. ${ }^{5}$ The goal is avoidance of hypertension. If an error is to be made it should be towards hypotension. Some advocate purposeful reduction of MAP during induction with vasodilatory agents (e.g., nitroprusside) to insure against hypertensive responses to intubation. This is usually unnecessary. Instead, controlled and progressive increases in depth of anesthesia sufficient to blunt responses to intubation are sufficient to prevent hemorrhage. This is an induction procedure that should not be rushed.

\section{Anesthesia maintenance}

Maintenance of anesthesia during craniotomy is usually uncomplicated and generic in many respects. There are 
two special considerations, however. In patients with intracranial mass lesions, brain bulk can be a problem, particularly when the dura is being opened. A swollen brain can herniate through the dural defect prohibiting further dural incision. In this circumstance the anesthesiologist is frequently requested to "relax" the brain. There are multiple methods by which this can be achieved. Usually several changes are made simultaneously which cumulatively result in improved operating conditions. The anesthesiologist can often prevent this problem during patient positioning by assuring the head is sufficiently elevated above the heart to promote venous drainage. Further head elevation intraoperatively can often cause dramatic reduction in brain bulk. This must be weighed against the risk of air embolism in which case transcardiac Doppler monitoring might be considered. This usually is not essential. Placement of the head 10-15 above the heart is usually sufficient to promote venous drainage without risk of air embolism or hemodynamic instability. ${ }^{6}$

Reduction in brain bulk can also be achieved by discontinuation of inhalation anesthetics that are known vasodilators. The first drug to discontinue is nitrous oxide. It is rapidly eliminated and a greater vasodilator than isoflurane because nitrous oxide does not have compensatory reduction in metabolic rate causing coupled reduction in cerebral blood flow $(\mathrm{CBF}) .^{7}$ Temporary discontinuation of the volatile anesthetic may also be of benefit.

There is no evidence that opioids increase brain bulk. There is evidence that opioids increase ICP. ${ }^{8}$ This effect, however, is modest and transient. Human study has provided convincing evidence that opioids increase ICP as a result of effects on MAP. ${ }^{9}$ When autoregulation is intact, reduced MAP causes vasodilation and a concordant increase in cerebral blood volume (CBV) and ICP. Opioid induced increases in ICP can be avoided simply by controlling MAP during opioid administration. As a result, opioids are unlikely to be an issue during maintenance with respect to brain bulk.

Mannitol reduces brain bulk by creating an osmotic gradient across the blood brain barrier causing water to flux from the extracellular extravascular to intravascular compartments. There also is evidence that mannitol improves deformability of red blood cells, thereby reducing viscosity promoting increased blood flow. As a result, autoregulation causes vasoconstriction that may reduce CBV. Mannitol is best given around time of skin incision (typically $0.5 \mathrm{mg} \cdot \mathrm{kg}^{-1}$ ) so the peak effect becomes available upon dural opening. Additional mannitol may be of value if the brain is still "tight".

Hyperventilation will be discussed below. The impact of hyperventilation on brain bulk is likely to be modest. Certainly, if hypercapnia is present it should be corrected. The response of the cerebral vasculature to changes in $\mathrm{PaCO}_{2}$ is rapid. There has been a major shift in attitude regarding the value of hypocapnia with the preponderance of opinion being that a major reduction in $\mathrm{PaCO}_{2}$ poses the risk of secondary ischemic injury. Stabilizing the $\mathrm{PaCO}_{2}$ in the range of $30-35 \mathrm{mmHg}$ is usually adequate.

If the above actions are not successful, some benefit may be obtained from administration of thiopental. Large doses cause major reduction in metabolic rate and a coupled reduction in CBF. This can be effective but will likely prohibit adequate neurologic evaluation upon emergence and also commit the patient to postoperative intubation and ventilation. It is suggested that consultation be made with the surgeon before taking this step.

Lumbar cerebrospinal fluid (CSF) drains are often placed for aneurysm surgery. The volume drained often exceeds $100 \mathrm{~mL}$ making this technique perhaps the most effective in reducing brain bulk of all options. The goal in aneurysm surgery, however, is different from that of tumour surgery. In most aneurysm cases, reduction in brain bulk is performed to reduce the magnitude of retractor pressure required to expose the aneurysm at the base of the brain. CSF drainage is usually not employed until after the dura is opened. This is because rapid and profound reduction in brain bulk can tear veins draining into sinuses. An acute subdural hematoma can be formed if the drain is opened prior to surgical exposure of the brain with little hope of prompt hemostasis. A practical approach to this is to ensure patency of the drain after positioning by observing progression of the air/fluid level through the connected catheter (usually less than 1 $\mathrm{mL}$ of CSF drainage is required to confirm this) and then leave the drain closed until surgical requirements dictate that it be opened. CSF drains are rarely used for most types of tumour surgery because of fear of transtentorial herniation. Occasionally CSF drains are placed for transphenoidal pituitary surgery, not to drain CSF, but to allow injection of saline or air to force the tumour towards the sphenoid sinus to facilitate surgical excision.

\section{Management of ventilation}

The classic reflex when confronting a patient with intracranial hypertension is use of hyperventilation. This is derived from knowledge that alteration of $\mathrm{PaCO}_{2}$, within the range of approximately 20-80 $\mathrm{mmHg}$, causes parallel changes in CBF. CBF, in fact, is only a surrogate for the true determinant of ICP, that being $\mathrm{CBV}$. $\mathrm{CBF}$ is easy to measure while $\mathrm{CBV}$ is not 
(particularly in humans). It is logical, however, that given a constant MAP, $\mathrm{PaCO}_{2}$ induced changes in $\mathrm{CBF}$ should correlate with cross-sectional diameter of the cerebral arterial vasculature. Decreasing $\mathrm{PaCO}_{2}$ results in decreased CBF and it is presumed that this also causes decreased CBV. Indeed, there is abundant clinical evidence in patients with ICP monitors in place, that reduction of $\mathrm{PaCO}_{2}$ results in at least transient reduction in ICP. Neuroanesthetic practice, therefore, had been to cause large reductions in $\mathrm{PaCO}_{2}$. Data from head injury patients has caused a major change in this perspective. Use of retrograde jugular venous hemoglobin- $\mathrm{O}_{2}$ saturation measurement techniques has repeatedly shown that reduction in $\mathrm{PaCO}_{2}$, in fact, can exacerbate cerebral hypoperfusion. ${ }^{10}$ This also makes common sense. If the problem with intracranial hypertension is decreased blood flow, it does not seem logical to treat the disorder with vasoconstriction. As a result, it is no longer advocated that major reductions in $\mathrm{PaCO}_{2}$ be made in patients undergoing craniotomy for space occupying lesions. Modest reductions in $\mathrm{PaCO}_{2}$ remain valuable, however, to counteract vasodilatory effects of volatile anesthetics.

It is also important to recognize the value of endtidal $\mathrm{CO}_{2}$ monitoring during craniotomy. This advance has reduced the need for repeated arterial blood gas sampling. However, analysis of arterial to end-tidal $\mathrm{CO}_{2}$ gradients in neurosurgical patients has shown that the gradient is not always predictable and should be measured for the individual patient when management of intracranial hypoperfusion is a concern.

\section{Muscle relaxants}

Several muscle relaxants have received special consideration in the context of craniotomy. The most interesting is succinylcholine. There is clear evidence from both experimental animals and humans that succinylcholine can increase ICP under conditions of intracranial hypertension. The magnitude of increase is typically small and transient. The mechanism was originally thought to be attributable, not to succinylcholine, but rather preservatives used in its formulation. This argument was dispelled. It has been shown in humans that ICP changes caused by succinylcholine can be blocked by pre-administration of a defasciculating dose of non-depolarizing relaxants. ${ }^{11}$ This suggests that fasciculations resulting from succinylcholine play a role in the ICP effects of this drug. Solid animal evidence supports this. A probable mechanism is the massive fasciculation-induced afferent barrage from muscle spindles to the brain that cause transient increases in metabolic rate and coupled increases in $\mathrm{CBF}^{12}$ Common sense plays a major role in the decision whether to use succinylcholine in patients with intracranial hypertension. Pretreatment with a small dose of a non-depolarizing agent most likely makes the argument moot. At the same time, emergency airway management and the clear desire to minimize hypercapnia and hypoxemia in patients with traumatic brain injury (TBI) dictate that succinylcholine can be an appropriate adjunct for tracheal intubation until a relaxant with similar speed of onset and duration of action is introduced to clinical practice.

There is clear evidence that the duration of action of non-depolarizing muscle relaxants is reduced by a variety of anti-convulsant medications. ${ }^{13}$ Even short durations of exposure to anti-convulsants can elicit this change. The mechanism for this remains unclear. Most patients requiring craniotomy are being treated with anticonvulsants. As a result, the non-depolarizing relaxant dosing regimen will, probably, require alteration. Atracurium and cis-atracurium seem to be largely resistant to these effects, most likely because metabolism is achieved by Hoffman elimination.

\section{Posterior fossa considerations}

Because of the primacy of the brainstem in maintaining vital function, posterior fossa procedures present special concerns. This is principally derived from three factors. First, the volume of the infratentorial compartment is small. Thus, smaller volumes of hematoma formation may be sufficient to compromise neural function. Further, brainstem edema developing after conclusion of surgery, may insidiously impair vital function. For this reason, it is appropriate to consult with the surgeon prior to planning extubation to determine if sufficient brain stem manipulation occurred such that concerns over brainstem edema warrant overnight endotracheal intubation. In this circumstance, a transient emergence from anesthesia in the operating room is often requested so as to allow definition of postoperative baseline motor function prior to giving sedation required to tolerate the endotracheal tube postoperatively. Second, retraction on the brainstem can cause loss of function of nuclei that regulate hemodynamics and ventilation. In earlier days of neurosurgery, infratentorial procedures were often performed with spontaneous ventilation to continuously monitor function of respiratory drive centres. This has largely been abandoned in favour of mechanical ventilation allowing control of brain bulk. A surrogate marker is heart rate. Precipitous decreases in heart rate are considered to be a signal of brain stem ischemia. This requires prompt notification of the surgeon. Most often, this clears spontaneously with adjustment of retractor placement. Occasionally, resuscitation with atropine is required. Third, cranial nerve function may be compromised. This is especially critical for the 
9th and 10th nerves that regulate gag reflex and laryngeal function. Again, consultation with the surgeon is helpful in defining potential for cranial nerve dysfunction. In such cases, it is appropriate to plan emergence such that integrity of the gag reflex can be tested prior to extubation. Absence of the gag reflex provides sufficient reason for continued intubation to prevent aspiration. In such cases, major sedation is usually unnecessary simply because the patient doesn't perceive the stimulation normally caused by the tube.

\section{Management of temperature}

There has been abundant enthusiasm over the past decade for routine use of intraoperative hypothermia in patients requiring brain surgery. This is based on overwhelming laboratory evidence that reduction of body temperature by even $2-3^{\circ} \mathrm{C}$ can cause major neuroprotection. ${ }^{14}$ Several problems remain before strong advocacy can be made for routine practice of induced hypothermia. First, there is virtually no evidence of mild hypothermia efficacy in humans. An appropriately powered trial in patients (with TBI) failed to define benefit and in fact identified worsened outcomes in the elderly. ${ }^{15}$ Despite considerable effort, there remains no clear evidence that induced hypothermia is neuroprotective in cardiac surgical patients. Finally, there is increasing evidence that mild hypothermia poses a variety of risks to the general surgical population. The International Hypothermia Aneurysm Surgery trial is currently being conducted with 1,000 patients being randomized to normothermia $\left(36.0-37.0^{\circ} \mathrm{C}\right)$ or hypothermia $\left(32.5-33.5^{\circ} \mathrm{C}\right)$ during intracranial aneurysm surgery. ${ }^{16}$ The results of this study will likely be available in 2003 . Until that study is completed, routine use of induced hypothermia for neurosurgical patients remains a speculative intraoperative intervention.

In contrast, there is near universal agreement that hyperthermia should be avoided. Not only is there absence of theoretical benefit, but substantial circumstantial evidence exists in humans that hyperthermia increases brain damage. Most studies supporting this contention are correlative and the data have not been obtained from a randomized trial. Such a trial is unlikely to be performed. It is also clear that hyperthermia is a frequent sequel to brain injury. ${ }^{17}$ Patients suffering from subarachnoid hemorrhage, TBI, or cardiac arrest are likely to have multiple spontaneous episodes of hyperthermia during acute convalescence. Vigilance and prompt treatment of hyperthermia is recommended.

\section{Pharmacologic neuroprotection}

A holy grail of academic neuroanesthesia has been the demonstrtion of neuroprotective efficacy of anesthetics and other purported neuroprotective compounds. The simple interpretation of four decades of research is that we still lack definitive proof (particularly in humans) that pharmacologic neuroprotection is a reality. Because most anesthetics reduce brain metabolic requirements, it certainly makes sense that in the context of diminished substrate supply anesthetics will increase tolerance to ischemia. However, the issue is now recognized to be far more complex than this logic. More important, other than a few studies examining barbiturates in cardiac surgical patients, there are no human studies that have prospectively defined presence or absence of neuroprotection from anesthetics.

Laboratory studies have now irrefutably shown that anesthetics increase tolerance of brain to ischemia. This is independent of the type of ischemia (global $v s$ focal). The mechanisms are likely related to inhibition of glutamatergic (excitatory) neurotransmission and potentiation of GABAergic (inhibitory) neurotransmission. Many anesthetics meet these criteria (i.e., volatile agents, propofol, barbiturates). Other compounds such as nitrous oxide and opioids appear to be inert. It is clear that animals sustaining stroke while awake have larger resultant lesions than if anesthetized. But which anesthetic is superior to another remains controversial. Many clinicians persist in use of thiopental as a first line agent. The logic for this is that thiopental has the longest track record of experimental efficacy and there is one human cardiac surgical trial that found benefit (albeit small). It is difficult, therefore, to recommend one agent as being superior to the others. There is one exception to this conclusion and that is etomidate. Although outcome evidence is not available, human studies have provided reasonable evidence that etomidate may exacerbate injury. ${ }^{18}$

Another problem is that if one selects an anesthetic to provide intraoperative neuroprotection the maximally efficacious dose remains undefined. There is surprising little laboratory data to guide this discussion. Most outcome studies are designed to compare different anesthetics without completion of dose-response curves for the respective anesthetics. Many would say that for barbiturates, maximal effect is obtained coincident with the dose required to provide EEG burst suppression. This is an anachronistic practice based on theoretical information. Recent studies in rodents indicate that substantially lower doses of barbiturates provide a similar magnitude of protection. ${ }^{19}$

As a result of incomplete science regarding anesthetic efficacy in humans, and in the absence of any other drugs approved for the purpose of neuroprotection in humans, the anesthesiologist is left with a speculative practice when providing pharmacologic 
neuroprotection. Perhaps the best we can do for the patient, with certainty, is to provide oxygenated blood at a sufficient perfusion pressure with simultaneous control of temperature and glucose concentration.

\section{Management of emergence}

Planning for emergence from anesthesia for craniotomy begins with anesthetic induction. The goals of emergence are a predictable recovery to allow testing of motor function in the context of controlled hemodynamics and airway. A unique concern is that failure to emerge may be attributable to either anesthesia or surgery. The treatment is highly dependent on the etiology. If failure to emerge is attributable to surgery, a computerized tomographic scan is usually performed to rule out hematoma formation. In contrast, if the problem is anesthesia based, patience in allowing elimination of anesthetic agents (or use of opioid antagonists or reversal of neuromuscular blockade) is the solution and the surgeon should be counselled that anesthesia is a likely explanation. Therefore, when planning the anesthetic, it is helpful to restrict use of agents to those that can be monitored for concentration or those for which sufficient knowledge of pharmacodynamics allows highly probable clearance by the time emergence is desired. As an example, induction doses of thiopental or propofol are unlikely to relate to failure to emerge from a three- to four-hour procedure. In contrast, persistent blood propofol concentrations sufficient to prevent awakening after a prolonged infusion may be difficult to diagnose. It is best to keep the anesthetic simple so that each compound can be independently ruled out as an etiology for failure to emerge.

Emergence from anesthesia for craniotomy presents several unique management concerns. It should be remembered that craniotomy is often performed in awake patients causing minimal discomfort. Thus, during most phases of surgery, the magnitude of surgical stimulation is also minimal. As a result, one of the strongest stimuli occurring during the procedure is application of the head dressing which causes sustained motion of the endotracheal tube. This combined with lightening of anesthesia for the anticipated emergence can result in loss of control of hemodynamics and difficulty in airway management, particularly if neuromuscular blockade is insufficiently reversed to allow extubation. A practical way to approach this event is to assume that the anesthesiologist has five to ten minutes after completion of the head dressing to allow a controlled emergence. Thus neuromuscular blockade is maintained until completion of the head dressing. Elimination of volatile anesthetics can be initiated at the time of bone flap replacement. Anesthesia is maintained by either residual concentration of opioid (i.e., fentanyl or sufentanil) or continued infusion of remifentanil. Supplementation with nitrous oxide is likely superior over use of $i v$ agents because its concentration can be defined by end-tidal gas analysis which aids in defining failure to emerge. Rapidly cleared iv agents such as lidocaine can be of value in sustaining anesthesia for a few additional minutes. If remifentanil is used, the rate of infusion can remain unchanged until the dressing is complete. ${ }^{20}$ This supports anesthesia during placement of the dressing but still allows a prompt and reliable emergence. It is important, however, to provide transitional analgesia before discontinuation of remifentanil. Administration of $10 \mathrm{mg}$ morphine or 100-150 $\mu \mathrm{g}$ fentanyl (in adults) is usually sufficient to provide analgesia without altering predictability of emergence.

For reasons not yet understood, patients undergoing craniotomy may exhibit hypertension during emergence that is sustained through the early phases of recovery. Because of the implications of intracranial hemorrhage, it is imperative to plan for treatment of hypertension before it becomes manifest. Prophylactic doses of labetolol are helpful, usually requiring 40-60 $\mathrm{mg}$ to be effective. It has not been proven that emergence hypertension contributes to hematoma formation. It has been shown, however, that many patients who develop postoperative hematomas have had episodes of hypertension during emergence or early recovery. ${ }^{21}$ The source of hemorrhage is almost always within the surgical field and thus quality of hemostasis undoubtedly is important. However, because the mortality associated with postoperative hematoma formation requiring emergent evacuation is high, it seems incumbent on the anesthesiologist to seriously attend to management of hemodynamics during emergence.

Postoperative nausea and vomiting (PONV) is a frequent problem after craniotomy. ${ }^{22}$ Several studies have shown that greater than $50 \%$ of patients suffer this complication. The incidence of PONV appears to be independent of whether the craniotomy was performed awake or under anesthesia, and independent of opioid dose. This suggests that surgery itself is contributory. Females, younger patients, and those undergoing infratentorial craniotomy are at greater risk. PONV is not only an early emergence problem but can be sustained for hours or days after surgery. Ample evidence is now available that prophylactic anti-emetic therapy markedly reduces the magnitude of this problem. Droperidol $(0.625 \mathrm{mg})$ appears to be at least as effective as $4 \mathrm{mg}$ ondansetron without causing detectable sedation. ${ }^{23}$ In either case, the duration of 
action of a single dose just prior to emergence is expected to be several hours. This is likely to be transiently beneficial and will require repeated supplementation if PONV is to continue to be suppressed.

\section{Emergency procedures}

It is important to know if the patient is dying of intracranial hypertension. This is particularly true of expanding hematomas. These cases are unquestionable surgical emergencies. The cure is surgical and the most important thing the anesthesiologist can do is make the patient ready for incision as rapidly as possible. Regardless of academic discussion on the importance of anesthetic effects on ICP in scheduled craniotomies, most agree, that in these patients all effort should be made to minimize increases in ICP. Patients who are herniating, or near herniating, are not likely to be conscious. Prevention of recall is of minimal concern. Effort should be made to secure the airway as rapidly as possible and turn the patient over to the surgeon. Small doses of barbiturate (so as to not cause hypotension) to reduce metabolic rate and coupled blood flow or opioid may be appropriate until surgical decompression is achieved. Profound hyperventilation should be avoided. Inhalational anesthetics have little or no role in the early stage of these procedures. Enhanced venous and arterial access can be made by the anesthesia team simultaneous with onset of surgery.

\section{References}

1 Jennett WB, McDowall DG, Barker J. The effect of halothane on intracranial pressure in cerebral tumors: report of two cases. J Neurosurg 1967; 26: 270-4.

2 Todd MM, Warner DS, Sokoll MD, et al. A prospective comparative trial of three anesthetics for supratentorial craniotomy: fentanyl/propofol, isoflurane/ $\mathrm{N} 2 \mathrm{O}$, and fentanyl/N2O. Anesthesiology 1993; 78: 1005-20.

3 Adams RW, Cucchiara RF, Gronert GA, Messick JM, Michenfelder JD. Isoflurane and cerebrospinal fluid pressure in neurosurgical patients. Anesthesiology 1981; 54: 97-9.

4 Gupta S, Heath K, Matta BF. Effect of incremental doses of sevoflurane on cerebral pressure autoregulation in humans. Brit J Anaesth 1997; 79: 469-72.

5 Tsementzis SA, Hitchcock ER. Outcome form "rescue clipping" of ruptured intracranial aneurysms during induction of anesthesia and endotracheal intubation. J Neurol Neurosurg Psych 1985; 48: 160-3.

6 Feldman Z, Kanter MJ, Robertson CS, et al. Effect of head elevation on intracranial pressure, cerebral perfusion pressure, and cerebral blood flow in head-injured patients. J Neurosurg 1992; 76: 207-11.

7 Lam AM, Mayberg TS, Eng CC, Cooper JO, Bachenberg
KL, Mathisen TL. Nitrous oxide-isoflurane anesthesia causes more cerebral vasodilation than an equipotent dose of isoflurane in humans. Anesth Analg 1994; 78: $462-8$.

8 Marx W, Shah N, Long C, et al. Sufentanil, alfentanil, and fentanyl: impact on cerebrospinal fluid pressure in patients with brain tumors. J Neurosurg Anesth 1989; 1: 3-7.

9 Werner C, Kochs E, Bause H, Hoffman WE, Schulte am Esch J. Effects of sufentanil on cerebral hemodynamics and intracranial pressure in patients with brain injury. Anesthesiology 1995; 83: 721-6.

10 Sheinberg M, Kanter MJ, Robertson CS, Contant CF, Narayan RK, Grossman RG. Continuous monitoring of jugular venous oxygen saturation in head-injured patients. J Neurosurg 1992; 76: 212-7.

11 Minton MD, Grosslight K, Stirt JA, Bedford RF. Increases in intracranial pressure from succinylcholine: prevention by prior nondepolarizing blockade. Anesthesiology 1986; 65: 165-9.

12 Lanier WL, Iaizzo PA, Milde JH. The effects of intravenous succinylcholine on cerebral function and muscle afferent activity following complete ischemia in halothane-anesthetized dogs. Anesthesiology 1990; 73: 485.

13 Ornstein E, Matteo R, Schwartz A, Silverberg P, Young $W$, Diaz J. The effect of phenytoin on the magnitude and duration of neuromuscular block following atracurium or vecuronium. Anesthesiology 1987; 67: 191-6.

14 Ginsberg MD, Sternan LL, Globus MT, Dietrich WD, Busto $R$. Therapeutic modulation of brain temperature - relevance to ischemic brain injury. Cerebrovasc Brain Metab Rev 1992; 4: 189-225.

15 Clifton GL, Miller ER, Choi SC, et al. Lack of effect of induction of hypothermia after acute brain injury. New Engl J Med 2001; 344: 556-63.

16 Hindman BJ, Todd MM, Gelb AW, et al. Mild hypothermia as a protective therapy during intracranial aneurysm surgery: a randomized prospective pilot trial. Neurosurgery 1999; 44: 23-33.

17 Albrecht RF, Wass CT, Lanier WL. Occurrence of potentially detrimental temperature alterations in hospitalized patients at risk for brain injury. Mayo Clin Proc 1998; 73: 629-35.

18 Hoffman WE, Charbel FT, Edelman G, Misra M, Ausman JI. Comparison of the effect of etomidate and desflurane on brain tissue gases and $\mathrm{pH}$ during prolonged middle cerebral artery occlusion. Anesthesiology 1998; 88: 1188-94.

19 Warner DS, Takaoka S, Wu B, et al. Electroencephalographic burst suppression is not required to elicit maximal neuroprotection from pento- 
barbital in a rat model of focal cerebral ischemia. Anesthesiology 1996; 84: 475-84.

20 Balakrishnan G, Raudzens P, Samra SK, et al. A comparison of remifentanil and fentanyl in patients undergoing surgery for intracranial mass lesions. Anesth Analg 2000; 91: 163-9.

21 Basali A, Mascha EJ, Kalfas I, Schubert A. Relation between perioperative hypertension and intracranial hemorrhage after craniotomy. Anesthesiology 2000; 93: 48-54.

22 Fabling JM, Gan TJ, Guy J, Borel CO, El-Moalem H, Warner DS. Postoperative nausea and vomiting: a retrospective analysis in patients undergoing elective craniotomy. J Neurosurg Anesth 1997; 9: 308-12.

23 Fabling JM, Gan TJ, Guy J, et al. A randomized, double-blind comparison of ondansetron, droperidol and placebo for prevention of post-operative nausea and vomiting after supratentorial craniotomy. Anesth Analg 2000; 91: 358-61. 